\title{
Fazari's Sign in Ultrasound Scan of Morbid Adherent Placenta/Placenta Accreta Spectrum (MAP/PAS) and Fazari's Triad during (MAP/PAS) Surgery
}

\author{
Atif Bashir Eltayeb Fazari1,2*, Zeinabsadat Tabatabaei Hakim1 \\ ${ }^{1}$ Latifa Hospital Dubai Health Authority, Dubai, UAE \\ ${ }^{2}$ University of Medical Sciences \& Technology, Khartoum, Sudan \\ Email: *atiffazari@hotmail.co.uk
}

How to cite this paper: Fazari, A.B.E. and Hakim, Z.T. (2020) Fazari's Sign in Ultrasound Scan of Morbid Adherent Placenta/Placenta Accreta Spectrum (MAP/PAS) and Fazari's Triad during (MAP/PAS) Surgery. Open Journal of Obstetrics and $G y$ necology, 10, 599-603.

https://doi.org/10.4236/ojog.2020.1040053

Received: April 5, 2020

Accepted: April 23, 2020

Published: April 26, 2020

Copyright $\odot 2020$ by author(s) and Scientific Research Publishing Inc. This work is licensed under the Creative Commons Attribution International License (CC BY 4.0).

http://creativecommons.org/licenses/by/4.0/

\begin{abstract}
Morbid Adherent Placenta (MAP)/Placenta Accreta Spectrum (PAS) is a serious diagnosis which has a risk of complications. Ultrasound scan helps in early diagnosis and has great value in further confirmation and follow up. Observed new clinical sign and associated clinical triad are discussed here.
\end{abstract}

\section{Keywords}

Morbid Adherent Placenta (MAP)/Placenta Accreta Spectrum (PAS), Ultrasound Scan, Stair Step Sign, Fazari's Triad

\section{Clinical Opinion}

Morbid Adherent Placenta (MAP)/Placenta Accreta Spectrum (PAS) is one of the most morbid conditions obstetricians will encounter. The rate has dramatically increased in the last 20 years. The three forms of morbidly adherent placenta (MAP): placenta accreta, increta and percreta, present a significant obstetric challenge, at times resulting in life-threatening bleeding and/or peripartum hysterectomy [1].

Myometrial tissue trauma and scarring are the main predisposing factors resulting in both placenta previa and morbidly adherent placenta. This can be caused by multiple Caesarean sections; this risk rises as the number of prior Caesarean sections increases. Placenta accreta spectrum is not exclusively a con${ }^{\star}$ Corresponding author. 
sequence of Caesarean delivery. Other surgical trauma to the integrity of the uterine endometrium and/or superficial myometrium, such as dilatation and curettage and other surgical injuries to the myometrium: manual removal of the placenta, postpartum endometritis or myomectomy, has been associated with accreta placentation in subsequent pregnancies, as well as, assisted reproductive technology and maternal smoking [2].

MAP/PAS poses dramatic risk for massive obstetric hemorrhage along with serious complication such as consumption coagulopathy, multisystem organ failure, and maternal mortality. The surgical management choices may be considered according to available expertise, geographical and individual circumstances [3]. In addition, there is an increased risk for surgical complications such as injury to bladder, ureters, and bowel and the need for reoperation. Most women require massive blood transfusion; many require admission to an intensive care unit. As a result of urgent intervention, preterm delivery is one of the sequalae expected in many cases, and often ends up by admission to a neonatal care intensive care unit. Centers of excellence in MAP/PAS management improve outcomes where multidisciplinary expertise and experience take care of MAP/PAS patients. Such expertise may include maternal-fetal medicine, gynecologic surgery, gynecologic oncology, vascular, trauma and urologic surgery, transfusion medicine, intensivists, neonatologists, interventional radiologists, anesthesiologists, specialized nursing staff, and ancillary personnel.

Ultrasound scan and Doppler are first-line investigations with high sensitivity. Transabdominal ultrasound is non-invasive; however, it may be difficult to visualize the lower uterine segment. Transvaginal ultrasound and color Doppler may improve the diagnostic accuracy especially in patients with placenta previa as it allows detailed visualization. Recent studies looking at the use of MRI have not demonstrated superiority of this modality over transvaginal ultrasound [4].

An accurate prenatal diagnosis is required to reduce the risk of maternal/fetal morbidity and mortality. Ultrasonography is used routinely for diagnosis of MAP/PAS, although diagnostic criteria and accuracy are still subject to debate. Two-dimensional (2D) power Doppler ultrasound could represent a turning point for diagnosis of abnormal placentation. Magnetic resonance imaging (MRI) can be helpful when the placenta is difficult to visualize on ultrasound due to the patient's body habitus or to a posterior location of the placenta and rarely required.

A finding of placenta previa should elicit a detailed evaluation for MAP/PAS specially with history of previous cesarean section, including color Doppler imaging and a transvaginal ultrasound scan.

Placental Lacunae: Placental lacunae have been the most predictive ultrasound scan finding for MAP/PAS, and often appear to be parallel linear vascular channels extending from the placental parenchyma into the uterine wall musculature. Presence of lacunae has the highest sensitivity in the diagnosis of MAP/PAS from early second trimester period and increasing numbers of lacunae are associated with increased risk for MAP/PAS. 


\section{Abnormal Color Doppler Imaging Patterns}

In MAP/PAS numerous large blood vessels are often seen surrounding the myometrium, possibly caused by the invasion of surrounding structures. However, increased vascularity is seen in any MAP/PAS. Invasion can also create an irregular bladder wall with extensive associated vascularity.

Loss of Retroplacental Clear Space: A retroplacental hypoechoic line is usually seen with normal placentation; it is one of the more obvious findings at screening evaluation and should prompt a detailed evaluation for other ultrasound scan markers.

Reduced Uterine Wall Thickness: One of the predictors for MAP/PAS is reduce myometrium thickness less than $3 \mathrm{~mm}$ (as measured between the echogenic serosa and the retroplacental vessels).

Careful assessment of the case with full bladder in early third trimester will show the stair step appearance (Fazari's sign) (Figure 1 and Figure 2) which is seen quite common among many cases of MAP/PAS and reviewed ultrasound scan and clinical observation with reported of intraoperative findings where triad (Fazari's Triad) are strongly associated with stair step appearance in ultrasound scan:

1) Ballooning of the lower segment all around as it occupied by the whole placenta [Figure 3].

2) Complete loss of uterine wall replaced by vascular bed [Figure 3, Figure 4].

3) Badly adherent urinary bladder with penetrated space by the placenta and its vasculature.

In practice and during surgery as all cases managed surgically, those cases are:

1) Technically very difficult to manage.

2) High risk for bladder injury than other cases without this sign.

3) Long time of surgery.

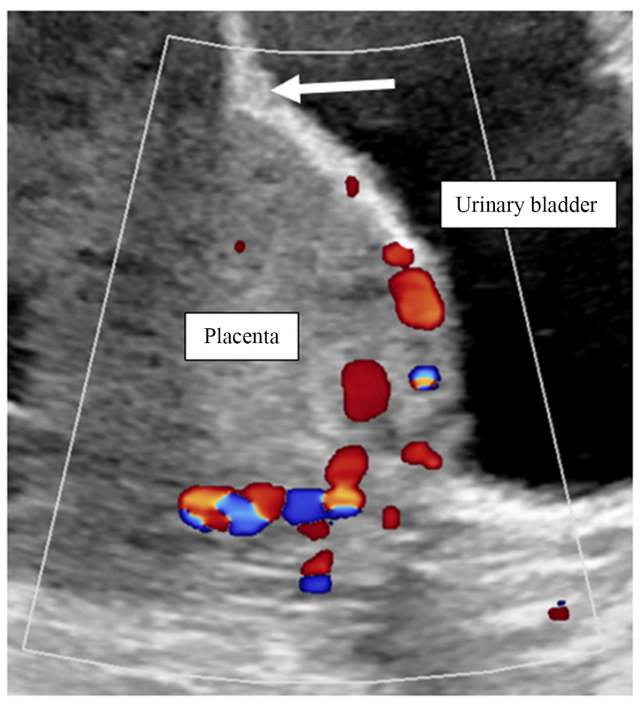

Figure 1. Sagittal ultrasound scan view shows the placenta with Doppler flow, urinary bladder wall with clear demarcation of the stair step sign (white arrow). 


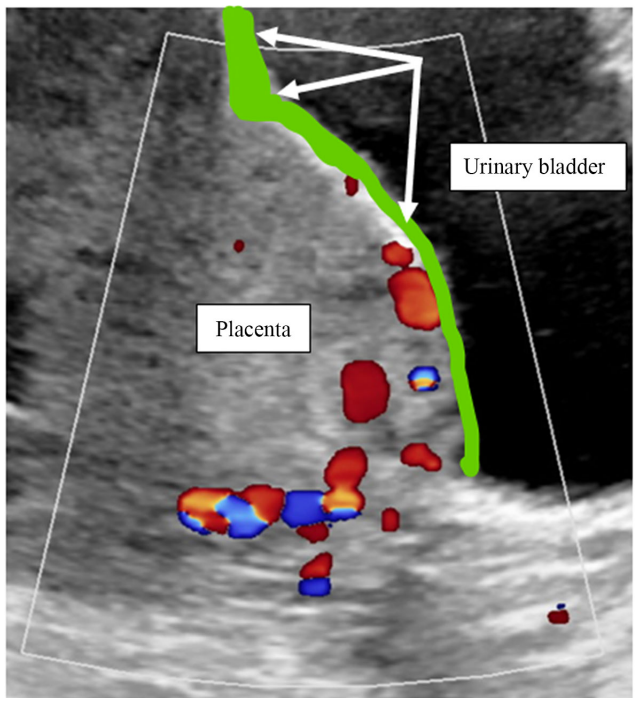

Figure 2. Sagittal ultrasound scan view shows stair step-Fazari sign marked with green line.

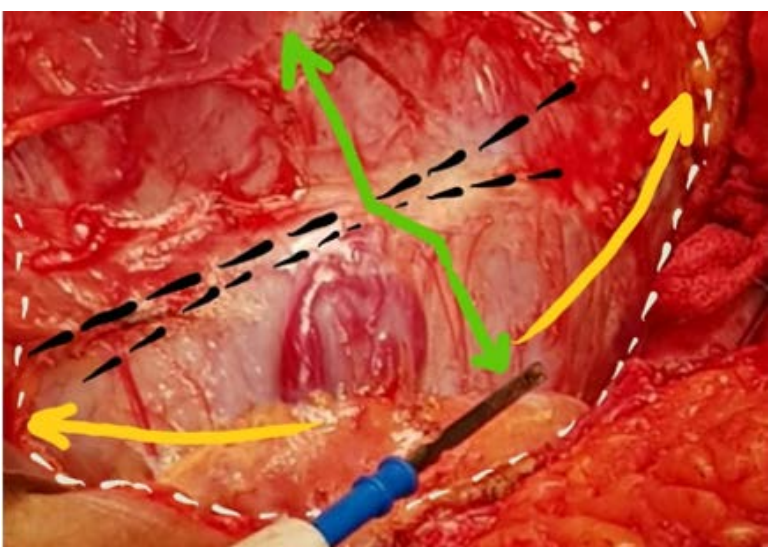

Figure 3. Intraoperative photo shows the ballooning of the lower segment all around as it occupied by the whole placenta (the yellow arrows and the white dotted area) with stair step (Green mark) over the released adhesion (above and below the black dotted lines) and vascular bed occupied the supposed wall.

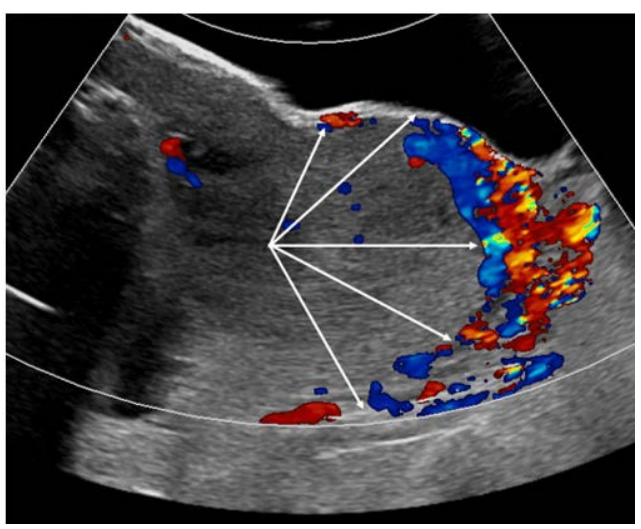

Figure 4. Coronal ultrasound scan view shows complete loss of uterine wall and replaced by vascular bed (white arrows). 
Anticipating the challenges and the outcome of each case of MAP/PAS by using the above-mentioned sign and triad, can prevent major complications that may occur and yield to good prognosis for the patient.

Being working with extensive experience in MAP/PAS since many years these clinical observations are quite proved. We do agree the number of MAP/PAS centers of excellence is still less than the needs; but sure, the clinicians are expert and doing great job in this field. Hence, considering the management of MAP/PAS, field of interest in subspecialty of high-risk obstetrics is of outmost importance for achieving the increase in the numbers of centers of excellence working with MAP/PAS. We do request to popularize this sign and the associated triad for further multi-centers study can take over for the sensitivity, specificity and the outcome.

\section{Conflicts of Interest}

The authors declare no conflicts of interest regarding the publication of this paper.

\section{References}

[1] Calì, G., Ggiambanco, L., Puccio, G. and Forlani, F. (2013) Morbidly Adherent Placenta: Evaluation of Ultrasound Diagnostic Criteria and Differentiation of Placenta Accreta from Percreta. Ultrasound in Obstetrics \& Gynecology, 41, 406-412. https://doi.org/10.1002/uog.12385

[2] Fazari, A., Aristondo, M., Azim, F., AlMaamari, B. and Eltayeb, R. (2019) Methotrexate in Management of Morbidly Adherent Placenta. Clinical Journal of Obstetrics \& Gynecology, 91-94.

[3] Babaei, M.R., Oveysi Kian, M., Naderi, Z., Khodaverdi, S., Raoofi, Z., et al. (2019) Methotrexate Infusion Followed by Uterine Artery Embolisation for the Management of Placental Adhesive Disorders: A Case Series. Clinical Radiology, 74, 378-383. https://doi.org/10.1016/j.crad.2019.01.006

[4] Jauniaux, E.R.M., Alfirevic, Z., Bhide, A.G., Belfort, M.A., Burton, G.J., et al. (2019) Placenta Praevia and Placenta Accreta: Diagnosis and Management. BJOG, 126, e1-e48. https://doi.org/10.1111/1471-0528.15306 